\title{
Confusing aspects of arrhythmogenic right ventricular dysplasia: Current clinical view
}

\author{
Melek Zekiye Ulucam
}

Department of Cardiology, Baskent University School of Medicine, Ankara, Turkey

Email: melekulucam@gmail.com

Received 11 January 2013; revised 14 February 2013; accepted 21 February 2013

\begin{abstract}
Arrhythmogenic right ventricular dysplasia is an inherited fatal cardiomyopathy causing fat and connective tissue infiltration of right ventricle. In advanced disease, it may also involve left ventricle. Heart failure, arrhythmias and sudden death are main clinical components. Many different genetic mutations can cause this cardiomyopathy. All mutations lead to the formation of the disease are not yet defined. Basis of pathogenetic event is corruption of physical connection as well as the electrical conduction between cardiomyocytes due to these genetic reasons. Genetical inheritance is frequently autosomal dominant but incomplete. Because of the many different phenotypes, there may be great heterogenity of clinical manifestations in same family. Diagnostic criteria are renovated lately. Current cardiac imaging techniques for the diagnosis are gradually progressing. A combination of several tests should be used for diagnosis. Early and accurate diagnosis will save lives and improve the patient's quality of life. In this paper, after mentioning the features and diagnosis of ARVD, differential diagnosis of ARVD from other diseases which may cause fatty infiltration of heart, arrhythmias and sudden death will be focused.
\end{abstract}

Keywords: Arrhythmogenic Right Ventricular Cardiomyopathy; Heart Failure; Arrhythmia; Sudden Death

\section{INTRODUCTION}

In recent years, with the contribution of the developments on diagnostic methods in patients with heart failure, major progress has been made in determining some of the rare and deadly diseases. One of them is the arrhythmogenic right ventricular dysplasia (ARVD), which it's importance has been better understood in recent years as an inherited cardiomyopathy. ARVD can lead heart failure, arrhythmias, and sudden death (Figure 1). Treatment with internal cardioverter defibrillator (ICD) sig- nificantly reduces mortality [1]. Heart transplantation may be required. There are many diseases that mimic some features of the ARVD in current clinical practice. Accuracy of differential diagnosis will accelerate the treatment process and dramatically reduce the morbidity and mortality of the disease.

\section{ARRHYTHMOGENIC RIGHT VENTRICULAR DYSPLASIA (ARVD)}

This genetic cardiomyopathy involves mainly the right ventricle (RV) but also rarely left ventricle (LV). It causes displacement of fatty and fibrous tissue of myocardium [2]. Involved regions consist a triangle of dysplasia, which RV subtricuspid area, apex, and the infundibulum are the corners. The direction of myocardial involvement begins at epicardium and progresses to endocardium. The result is localized or generalized fibrofatty infiltration of RV and sometimes LV myocardium. Structural abnormalities range from myocardial aneurysms to regional or global dysfunction. The incidence varies between 4/1000 - 1/10,000 [3].

Genetical inheritance is usually autosomal dominant but sometimes may be autosomal recessive. However, due to incomplete penetrance, age of onset of symptoms and the clinical picture may be very different among individuals with same genetic structure. All causative mutations are not clarified yet [4]. Due to these genetic reasons, physical connection as well as the electrical conduction between cardiomyocytes are corrupted, and this forms the basis of pathogenetic event.

Symptoms usually begins at 2nd or 3rd decade. Due to the wide distribution of the clinical phenotype, everyone is different and diagnosis is difficult. Early diagnosis and early treatment not only save the life, but improve the quality of life.

Clinical picture may be different for each patient $[5,6]$. The most common clinical presentation is characterized by heart failure, arrhythmias and sudden death. There are findings of right heart failure in varying degrees in almost every case. Patients may be asymptomatic or in 


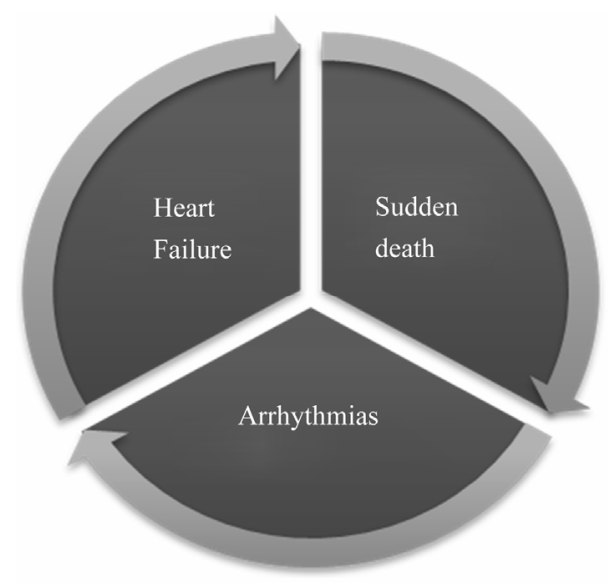

Figure 1. Main clinical components of Arrhythmogenic right ventricular dysplasia.

severe right heart failure. Ventricular arrhythmias are sensitive of catecholamines and exercise. Broad spectrum of arrhythmia may vary from ventricular premature pulses to ventricular tachycardia (VT) and ventricular fibrillation. Premature ventricular contractions has right axis and may be multifocal. VT is right ventricular outflow tract tachycardia with left bundle branch block. Incidence of sudden death reveals the importance of the disease dramatically. ARVD is responsible for $17 \%$ $20 \%$ of sudden cardiac death in young people. Annual sudden death rate is about $1 \%$ [4]. There is usually a history of syncope in cases of sudden death in the past.

Diagnostic criteria are renovated in 2010 [7]. Two major or (1 major +2 minor) criteria are required for "definitive diagnosis". This guide, defined "borderline" ARVD by ( 1 major +1 minor) or 3 minor criteria and "possible" ARVD by 1 major or 2 minor criteria for the first time.

Major and minor diagnostic criteria obtained from six categorical classes defined by 1-global/regional structural and functional change, 2-tissue characterisation, 3repolarization abnormalities, 4-depolarization/conduction abnormalities, 5-arrhythmias and 6-family history [7]. Global/regional structural and functional criteria detected by two-dimensional echocardiography, magnetic resonance imaging (MRI) and RV angiography. Tissue characterisation is determined by pathological tissue sections. Repolarization abnormalities diagnosed by ECG, depolarization and conduction abnormalities are diagnosed by ECG and signal-averaged ECG, arrhytmias determined by ECG. Diagnosis includes integration of information derived from medical history, physical examination, ECG, signal averaged ECG, echocardiography, 24-hour Holter monitoring, cardiac MRI, cardiac CT angiography, endomyocardial biopsy, RV angiography (specificity 90\%, the gold standard), and electrophysiological methods.

Echocardiography is the most commonly used diag- nostic test in ARVD. Major and minor findings may be determined and it is an easy applicable diagnostic tool. Findings can be summarized as dilatation of the RV outflow tract, RV systolic dysfunction, aneurysms, prominent trabeculations and moderator band [8]. In ARVD patients, tissue Doppler and two-dimensional deformation analysis of RV was found to be superior to two-dimensional echocardiography. In a study, optimal cut-off value for tissue Doppler strain value in any of the three segments (RV free wall, apical, mid and basal segments) was determined as $\mathbf{1 8 . 2 \%}$ for RV dysfunction in ARVD [9]. In another publication, even if RV were completely normal at conventional echocardiography, systolic velocity and displacement measurements at RV were significantly lower than controls. Cut-off values for RV dysfunction were $<7.5 \mathrm{~cm} / \mathrm{s}$ for peak systolic flow velocity and $<18 \%$ for peak RV strain [10]. Two dimensional echocardiography is not recommended for the the evaluation of RV volume, 3D TTE or 3D TEE is suggested method. In ARVD patients, it is shown that cardiac MRI is compatible with $3 \mathrm{D}$ echocardiography in the assessment of RV volume [11].

Echocardiography is not always enough for diagnosis of ARVD. Another major contribution to diagnosis is established by cardiac MRI. Functional findings in MRI are regional wall motion abnormalities, focal aneurysms, dilatation of the RV, RV systolic/diastolic dysfunction. Morphological MRI data are myocardial fatty infiltration, focal wall thinning, trabecular hypertrophy and disarray, hypertrophy of the moderator band and dilatation of RV outflow tract [12]. MRI gives comprehensive information about heart size, myocardial function, myocardial structure [Muscle, fat infiltration, fibrosis (gadolinium uptake increased in the late phase)] in ARVD. Limitations of the MRI assessment are being dependent of the sensitivity and specificity of practitioners, artifacts due to cardiac arrhythmias, bad images, limited use of MRI and presence of intracardiac devices. It is not a method which is possible to applicate everywhere and everyone [13]. CT angiography, can show almost same results with MRI. It can be preferred if cardiac MRI image quality is insufficient and in the presence of arrhythmias. It is advantageous in patients with cardiac device/prosthesis and being an easy applicable test. The radiation is the disadvantage of the method and may restrict the use of the most important feature [14].

In all the parameters studied up to date, RV angiography is considered as gold standard. Specificity is $90 \%$ for the diagnosis of ARVD. Diagnostic value of endomyocardial biopsy may contribute diagnosis, but data are frequently false negative [5]. If diagnosis is still not definite, diagnosis can be made by electrophysiological study and endocardial voltage mapping and typical myocardial scar can be diagnosed at early stage [15]. 


\section{DIFFERENTIAL DIAGNOSIS OF RIGHT VENTRICULAR DYSPLASIA}

\subsection{Disease with Fatty Infiltration of Myocardium}

The defining characteristic of ARVD is fatty infiltration of the RV myocardium. It can be best detected by MRI. Sensitivity and specificity for fatty infiltration, RV enlargement and regional RV dysfunction detected by MRI are: 84\%, 68\%, 78\% and 79\%, 96\%, 94\% respectively. Most sensitive, least specific and least reproducible finding is fatty infiltration. In reality, fatty infiltration of myocardium is a condition that may develop due to many reasons. It may be physiological related with aging, or may be pathologic due to ARVD or healed myocardial infarction and other reasons $[13,16]$.

For differential diagnosis of ARVD, reason for fatty infiltration should be explored. Typical fatty infiltration of myocardium in ARVD, usually involves RV free wall, RV outflow tract (RVOT), trabeculae of RV, moderator band, right side of the interventricular septum and LV free wall of young men. It is predominantly subepicardial. Myocardium is thin and right ventricle is dilated. Physiological fatty infitration of myocardium, usually associated with aging and women gender. Anterolateral free wall of RV, RV outflow tract, trabeculae of RV, the apex of the LV are frequently involved. All of the wall thickness is affected. As a result, myocardial thickness is normal or increased. Ventricular size is normal. Healed myocardial infarction is usually observed in middleaged/elderly cases. Infarct area, often involves LV and more pronounced at subendocardium. Myocardial thickness is normal or thin, RV is normal or dilated.

\subsection{Cardiomyopathies Causing Fibrosis of Myocardium and Sudden Cardiac Death}

In recent years, it is recognized that, there are some disease which cause fibrosis of myocardium like RV fibrosis in ARVD. They also cause arrhythmias and sudden death like ARVD.

Most important of them is known as left-dominant arrhythmogenic cardiomyopathy. It is characterized as fibroadipose tissue development involving right side of the interventricular septum and 1/3 thirds outer of LV wall. It is thought to be due to mutations in desmoplakin. Sometimes it is considered as 3rd form of ARVD remembering first form involves RV while 2nd form involves RV and LV. There are negative T vawes at inferolateral LV wall at ECG. It can lead to VT with RBBB morphology [17].

Idiopathic LV myocardial fibrosis is the cause of $1 \%$ $3 \%$ of sudden cardiac death in autopsy cases. It is characterized by heterogeneous interstitial fibrosis of the inferior wall of LV without coronary artery disease and other causes. The aetiology is uncertain. It may be related with aging or myocarditis. Some authors accept it as a member of the ARVD family. It's low prevalence may be due to it's nature as a difficult disease to diagnose and identify. If it's clinical and genetic profile would be better recognized, more cases will be dignosed [18].

Distinction of Uhl anomaly and ARVD is very important. In Uhl anomaly, right ventricular wall is thin as a paper, it contains only endocardium and epicardium. In ARVD patients, there is also fibrosis and fatty infiltration at the wall of the RV. Uhl anomaly begins at fetal life, it can be recognised by fetal echo. It is associated with the cyanosis, dyspnea, and right heart failure. ARVD is usually recognized in adolescence and characterized by arrhythmias. Uhl anomaly may accompany other congenital syndromes (tricuspid valve dysplasia, pulmonary atresia, PDA). Male/Female ratio is $1.27 / 1$ in Uhl anomaly and 2.28/1 at ARVD patients respectively. Pathological process is continuous in Uhl anomaly and episodic at ARVD [19].

\subsection{Arrhytmic Diseases of the Right Ventricle}

In clinical cases with arrhythmias caused by right ventricle, differential diagnosis of ARVD may be difficult.

Differential diagnosis of idiopathic RVOT tachycardia and ARVD is essential. Both of them can lead to tachycardia with left bundle branch block originating from the $\mathrm{RV}$, but they are different in terms of findings, clinical significance and treatment. Idiopathic RVOT tachycardia is seen in people without structural heart disease and respond to medical therapy and radiofrequency ablation. There is no history of arrhythmia and sudden death in the family. Arrhythmias range from premature ventricular contractions to VT. Sudden cardiac death is rare. T waves at ECG is normal, signal averaged ECG and MRI is usually normal [20]. In ARVD patients, sudden cardiac death may be seen and ICD therapy may be required. Structural abnormalities of RV are detected at cardiac imaging. In electrophysiological stimulation studies, arrhythmias are usually monomorphic at idiopathic RV outflow tract tachycardia, while polymorphic in ARVD patients. The most important thing to remember here is that, in patients with RV outflow tract tachycardia, ARVD should be remembered as a possible cause, because it may change our strategy of treatment. Echocardiographic findings in the diagnosis of ARVD is not always enough. If ARVD is suspected, and echocardiographic findings were normal, additional methods like the RV angiography and MRI should be done to make the diagnosis clear.

Another disease that can lead to arrhythmias and sudden death is Brugada syndrome. It is usually a structural disorder of the sodium channels. To make the distinction 
of ARVD and Brugada syndrome, we need to know that, Brugada syndrome and ARVD can be seen at any age, both of them more common in men. In Brugada syndrome, there is dynamic ECG changes, the ECG in ARVD patients has fixed abnormalities. Echo and angio is normal in Brugada syndrome and RV dilatation and dysfunction observed in ARVD patients. MRI and pathological findings are normal in Brugada syndrome and fatty infiltration and fibrosis are findings of ARVD patients. Brugada syndrome and ARVD may have common clinic (arrhythmias, sudden death) clues but there is heart failure in ARVD patients [21].

\section{TREATMENT OF RIGHT VENTRICULAR DYSPLASIA}

Main goal for the treatment of ARVD is to decrease the incidence of sudden cardiac death. Specific cases with ARVD are considered at high risk for sudden cardiac death. They include: young age, competitive sports activity, malignant familial history, extensive RV disease, biventricular involvement, syncope, episode of ventricular arrhythmia. Beyond these parameters, programmed electrical stimulation during electrophysiologic study may establish comprehensive prognostic information related with arrhytmogenic potential of ARVD before selection of the treatment method.

Treatment options are lifestyle modification, pharmacological therapy, catheter ablation, placement of an implantable cardioverter-defibrillator and cardiac transplant surgery. For lifestyle modification, rigorous physical activity and cardiac stimulants should be prohibited. Beta blockers, (especially sotalol and metoprolol) and amiodarone are best options for pharmacologic therapy. Angiotensin converting enzyme inhibitors can be given but beneficial effects for delaying progression of ARVD is not proven. In order to prevent thrombus formation in RV and pulmonary embolism, warfarin should be used. Catheter ablation is effective in $60 \%-90 \%$ of cases but recurrences are common. Indications are drug-refractory VT and frequent recurrence of VT after ICD placement. An ICD is the most effective prevention against sudden cardiac death but progression of the disease at the lead attachment point may cause sense/pace problems. Cardiac transplant surgery may be performed in arrhythmias refractory to treatment and progressive disease with severe biventricular heart failure despite all efforts for treatment.

\section{FURTHER RESEARCH TREND OF ARVD}

There are still many unanswered questions about the diagnosis and treatment of ARVD. For example, it is unclear how to treat borderline or possible ARVD, espe- cially in relatives of patients. Natural history of the borderline or possible ARVD should be better defined. More research is still needed to better define which patients will benefit from the placement of implantable cardioverter-defibrillators. More research should be done for the identification of unknown genes that cause ARVD. This information will facilitate the diagnosis of the disease, and illuminate genotype/phenotype relationships. In addition, the identification of the specific genetic defects may ultimately have specific therapeutic implications for gene therapy.

\section{REFERENCES}

[1] Dalal, D., Nasir, K., Bomma, C., Prakasa, K., Tandri, H., Piccini, J., et al. (2005) Arrhythmogenic right ventricular dysplasia: A United States experience. Circulation, 112, 3823-3832. doi:10.1161/CIRCULATIONAHA.105.542266

[2] Basso, C., Corrado, D., Marcus, F.I., Nava, A. and Thiene, G. (2009) Arrhythmogenic right ventricular cardiomyopathy. Lancet, 373, 1289-1300. doi:10.1016/S0140-6736(09)60256-7

[3] Calkins, H. (2008) Classification of the cardiomyopathies: A position statement from the European Society of Carology Working Group on myocardial and pericardial disses. European Heart Journal, 29, 270-276. doi:10.1093/eurheartj/ehm342

[4] Elliott, P., Andersson, B., Arbustini, E., Bilinska, Z., Cecchi, F., Charron, P., et al. (2008) Classification of the cardiomyopathies: A position statement from the European Society of Cardiology Working Group on myocardial and pericardial diseases. European Heart Journal, 29, 270-276. doi:10.1093/eurheartj/ehm342

[5] McKenna, W.J., Thiene, G., Nava, A., Fontaliran, F., Blomstrom-Lundqvist, C., Fontaine, G., et al. (1994) Diagnosis of arrhythmogenic right ventricular dysplasia/cardiomyopathy. Task Force of the Working Group Myocardial and Pericardial Disease of the European Society of Cardiology and of the Scientific Council on Cardiomyopathies of the International Society and Federation of Cardiology. British Heart Journal, 71, 215-218. doi:10.1136/hrt.71.3.215

[6] Fontaine, G. and Frank, R. (2006) Arrhythmogenic right ventricular dysplasia: 2006 update. Training for Audiovisual Preservation in Europe, 4, 99-107

[7] Marcus, F.I., McKenna, W.J., Sherrill, D., Basso, C., Bauce, B., Bluemke, D.A., et al. (2010) Diagnosis of arrhythmogenic right ventricular cardiomyopathy/dysplasia: Proposed mofification of the Task Force Criteria. European Heart Journal, 31, 806-814. doi:10.1093/eurheartj/ehq025

[8] Yoerger, D.M., Marcus, F., Sherrill, D., Calkins, H., Towbin, J.A., Zareba, W., et al. (2005) Multidisciplinary study of right ventricular dysplasia investigators. Echocardiographic findings in patients meeting task force criteria for arrhytmogenic right ventricular dysplasia: New insights from the multidisciplinary study of right ventri- 
cular dysplasia. Journal of the American College of Cardiology, 45, 860-865. doi:10.1016/j.jacc.2004.10.070

[9] Teske, A.J., Cox, M.G., De Boeck, B.W., Doevendans, P.A., Hauer, R.N. and Cramer, M.J. (2009) Echocardiographic tissue deformation imaging quantifies abnormal regional right ventricular function in arrhythmogenic right ventricular dysplasia/cardiomyopathy. Journal of the American Society of Echocardiography, 22, 920-927. doi:10.1016/j.echo.2009.05.014

[10] Prakasa, K.R., Wang, J., Tandri, H., Dalal, D., Bomma, C., Chojnowski, R., et al. (2007) Utility of tissue Doppler and strain echocardiography in arrhythmogenic right ventricular dysplasia/cardiomyopathy. American Journal of Cardiology, 100, 507-512. doi:10.1016/j.amjcard.2007.03.053

[11] Kjaergaard, J., Hastrup Svendsen, J., Sogaard, P., Chen, X., Bay Nielsen, H., Kober, L., et al. (2007) Advanced quantitative echocardiography in arrhythmogenic right ventricular cardiomyopathy. Journal of the American Society of Echocardiography, 20, 27-35. doi:10.1016/j.echo.2006.07.006

[12] Jain, A., Tandri, H., Calkins, H. and Bluemke, D.A. (2008) Role of cardiovascular magnetic resonance imaging in arrhytmogenic right ventricular dysplasia. Journal of Cardiovascular Magnetic Resonance, 10, 32. doi:10.1186/1532-429X-10-32

[13] Tandri, H., Castillo, E., Ferrari, V.A., Nasir, K., Dalal, D., Bomma, C., et al. (2006) Magnetic resonance imaging of arrhythmogenic right ventricular dysplasia: sensitivity, specificity, and observer variability of fat detection versus functional analysis of the right ventricle. Journal of the American College of Cardiology, 48, 2277-2284. doi:10.1016/j.jacc.2006.07.051

[14] Soh, E.K., Villines, T.C. and Feuerstein, I.M. (2008) Sixty-four-multislice computed tomography in a patient with arrhythmogenic right ventricular dysplasia. Journal of Cardiovascular Computed Tomography, 2, 191-192. doi:10.1016/j.jcct.2008.02.006

[15] Marchlinski, F.E., Zado, E., Dixit, S., Gerstenfeld, E.,
Callans, D.J., Hsia, H., et al. (2004) Elecroanatomic substrate and outcome of catheter ablative therapy for ventricular tachhycardia in setting of right ventricular cardiomyopathy. Circulation, 110, 2293-2298. doi:10.1161/01.CIR.0000145154.02436.90

[16] Kimura, F., Matsuo, Y., Nakajima, T., Nishikawa, T., Kawamura, S., Sannohe, S., et al. (2010) Myocardial fat at cardiac imaging: How can we differentiate pathologic from physiologic fatty infiltration? RadioGraphics, 30, 1587-1602. doi:10.1148/rg.306105519

[17] Sen-Chowdhry, S., Syrris, P., Prasad, S.K., Hughes, S.E., Merrifield, R., Ward, D., et al. (2008) Left-dominant arrhythmogenic cardiomyopathy: An under-recognized clinical entity. Journal of the American College of Cardiology, 52, 2175-2187. doi:10.1016/j.jacc.2008.09.019

[18] John, B.T., Tamarappoo, B.K., Titus, J.L., Edwards, W.D., Shen, W.K. and Chugh, S.S. (2004) Global remodeling of the ventricular interstitium in idiopathic myocardial fibrosis and sudden cardiac death. Heart Rhythm, 1, 141-149. doi:10.1016/j.hrthm.2004.02.021

[19] Pamuru, P.R., Dokuparthi, M.V., Remersu, S., Calambur, N. and Nallari, P. (2010) Comparison of Uhl's anomaly, right ventricular outflow tract ventricular tachycardia (RVOT VT) \& arrhythmogenic right ventricular dysplasia/cardiomyopathy (ARVD/C) with an insight into genetics of ARVD/C. Indian Journal of Medical Research, 131, 35-45.

[20] Tekin, M., Canbay, A. and Diker, E. (2009) Arrhythmogenic right ventricular cardiomyopathy mimicking right ventricular outflow tract tachycardia. Archives of the Turkish Society of Cardiology, 37, 566-568.

[21] Naccarella, F., Naccarelli, G., Fattori, R., Nava, A., Martini, B., Corrado, D., et al. (2001) Arrhythmogenic right ventricular dysplasia: Cardiomyopathy current opinions on diagnostic and therapeutic aspects. Current Opinion in Cardiology, 16, 8-16. doi:10.1097/00001573-200101000-00002 